\title{
Classification Characteristics of a Cyclone Type Classifier with Improved Collection Boxes for Separating Particles near the Wall Surface
}

\author{
Teruo Oshitari ${ }^{1,2}$, Kazumi Yamamoto ${ }^{3}$, Kunihiro Fukui ${ }^{1}$ and \\ Hideto YoshidA ${ }^{1}$ \\ ${ }^{1}$ Department of Chemical Engineering, Hiroshima University, 1-4-1 Kagamiyama, \\ Higashi-Hiroshima-shi, Hiroshima 739-8527, Japan \\ ${ }^{2}$ Soken Chemical \& Engineering Co., Ltd., 3-29-5 Takada, Toshima-ku, Tokyo 171-8531, Japan \\ ${ }^{3}$ Soken Chemical \& Engineering Co., Ltd., 1-13-1 Hirosehigashi, Sayama-shi, Saitama 350-1320, Japan
}

\begin{abstract}
Keywords: Gas-Cyclone, Particle Separation, Cut-Off Size, Classification Effectiveness, Sharpness Index
Cyclone separators are used in many processes to remove powders for collection in a wide range of diameters. When the cyclone separator is used as a classification device that requires high effectiveness, the $50 \%$ cut-off size $\left(D_{\mathrm{p} 50}\right)$ is restricted to less than several microns owing to the strong centrifugal force. We developed a cyclone-type classifier to effectively classify coarse particles collected by the normal cyclone, as discussed in our previous study. The classifier comprises inner and outer collection boxes with a sloping channel region between the upper sections. The sloping channel could derive the additional air, called blow-up flow, from the tangent nozzle established in the outer box. The blow-up flow becomes confluent with the main swirling flow of the cyclone. In the channel, the fine particles affected by the drag of the blowup flow were collected in the inner box. Additionally, the coarse particles affected by the centrifugal force induced by the swirling flow were collected in the outer box. The previous study, regarding a classifier with a channel length of $6 \mathrm{~mm}$, demonstrated that $D_{\mathrm{p} 50}$ and the effectiveness of the classification $\kappa$, which is the ratio of the $25 \%$ cut-off size to the $75 \%$ cut-off size $\left(D_{\mathrm{p} 25} / D_{\mathrm{p} 75}\right)$, increase linearly with the increase in blow-up flow rate $(\Delta q)$. In this study, we increased the channel length from 6 to $23 \mathrm{~mm}$. This resulted in the following beneficial characteristics for separating acrylic particles having a size distribution of $15-35 \mu \mathrm{m}$. Unlike the previous study, $D_{\mathrm{p} 50}$ depended on the main flow rate at the entrance regardless of $\Delta q$ since the increase in $\Delta q$ increases the centrifugal force, which acts simultaneously on the $D_{\mathrm{p} 50}$ particles, and the drag force. Meanwhile, $\boldsymbol{k}$ increased significantly with the increase in $\Delta q$. The classifier classified the particles with high effectiveness ( $k$ ranging from 0.75 to 0.92 ).
\end{abstract}

\section{Introduction}

Cyclone separators possess advantages such as a simple structure and are maintenance free since they have no drives or rotors. The swirling flow is so strong that particles in the air are effectively collected in the collection box due to the inertial force differences. Therefore, the cyclone separator is widely used as a general-purpose collection device in many processes for producing powders such as those of ceramics, inorganic material, metals, resin, food, and toners.

Meanwhile, cyclones have been studied for a $50 \%$ cutoff size termed $D_{\mathrm{p} 50}$, the collecting efficiency after an examination of the velocity distribution, and the pressure loss within a cyclone separator. Barth (1956) proposed a numerical model for predicting $D_{\mathrm{p} 50}$ called the Equilibrium-Orbit Model, which depends on a balance between drag and centrifugal forces acting on a particle on a control surface (CS). Barth considered the cylindrical CS that is formed by continuing the vortex finder wall to the bottom of the cyclone.

Received on September 13, 2016; accepted on December 22, 2016 DOI: 10.1252 /jcej.16we267

Correspondence concerning this article should be addressed to $\mathrm{T}$. Oshitari (E-mail address: oshitari@soken-ce.co.jp).
He estimated the tangential velocity over the CS using the wall velocity as calculated from the inlet gas velocity. These estimations are dependent on the friction factor. Hoffmann and Stein (2008) provided the friction factor that included the influence of the particle laden air flow over a hydraulically smooth cyclone surface. Furthermore, Muschelknautz and Trefz (1990) developed Barth's model in greater detail. They suggested a tangential velocity for the gas at the inner core radius of the CS using a friction factor coefficient.

The coefficient was related to the defined Reynolds number for a cyclone body when taking wall roughness into consideration. These models give the basic method for predicting $D_{\mathrm{p} 50}$ in the field of a swirling flow in a classification device.

Cortés and Gil (2007) reviewed many past studies on the cyclone separator and examined various models for formulating the velocity distributions and pressure loss. The tangential velocity profile in the cyclone was described as a Rankine vortex combined with forced and free vortices. They proposed the use of the Reydon and Gauvin (1981) model for the accurate estimation of the tangential velocity profile with the forced vortex, and the Meissner and Loffler (1978) model for that of the same for the free vortex. Hoffmann and Stein (2008) also investigated many past studies 
and provided various design techniques for applying cyclone separators to practical processes.

However, the common purpose of these studies was to collect particles, and not to classify them. Generally, when cyclone separators are used as classification devices requiring high effectiveness and flexibility of the cut-off size, the critical diameter is less than several microns for a strong swirling flow in the cyclone separator. The studies in this range of diameters are discussed here. Iinoya et al. (1992) extended the cut-off size to the submicron level and improved the classification effectiveness by using the blowdown method. Yoshida et al. (1995) tracked the behavior of the particles in a blow-down cyclone separator using computational fluid dynamics (CFD), and explained the mechanism by which the particle cut-off size became small. Yoshida et al. (1993) could control the cut-off size to be within the range of $0.45-0.75 \mu \mathrm{m}$ by installing a movable inlet guide plate that allowed the inlet clearance to change in the cyclone entrance section.

Akiyama et al. (2005) lowered the cut-off size and improved the classification effectiveness by increasing the flow rate through the nozzle attached to the top section of the cyclone separator. Additionally, Takeda et al. (2014) recently suggested a method to lower the cut-off size while controlling the pressure loss by introducing clean air from an entrance nozzle installed at the inlet guide plate in the cyclone separator. These studies developed methods, by which the tangential velocity in the vicinity of the cyclone wall can be increased, to ensure that a strong centrifugal force acts on a particle and results in improved classification effectiveness.

These studies focused on the classification of particles less than several microns as described earlier. There are no reports that study the classification of coarse particles more than several microns. When the cut-off size is intended to be raised to more than several microns for a conventional cyclone separator, the swirling flow must be weakened. This weakening reduces the centrifugal force acting on the particle and leads to low classification effectiveness. Additionally, the lowered centrifugal force narrows the range of activities where the cyclone separators can be applied as classification devices.

Oshitari et al. (2015) modified a conventional cyclone to install a CS on the wall where particles passing the wall surface were classified. The classifier comprised inner and outer collection boxes having a sloping channel region between the upper sections of the boxes. The channel can derive additional air, called blow-up flow, from the tangent nozzle that is installed in the outer box, thereby creating a confluent flow with the main swirling flow of the cyclone separator. This type of a cyclone separator classifier can utilize the strong swirling flow and establish a wider range of the cut-off size $D_{\mathrm{p} 50}$ than that by the conventional cyclone separators. It is expected that the modified cyclone will be a versatile classification device and can be widely applied to the production of various kinds of powders.

In this study, we designed the channel to be $23 \mathrm{~mm}$ and performed classification of acrylic particles using the same diameter distribution $(15-35 \mu \mathrm{m})$ as the one in our previous study. However, in the previous study (Oshitari et al., 2015), the channel was designed to be $6 \mathrm{~mm}$. The classifier showed excellent operational characteristics and was unlike the one in the previous study. The effectiveness of the classification improved as did the yield without significantly changing $D_{\mathrm{p} 50}$. The purpose of this study was to clarify the classification mechanism by examining the cut-off size $D_{\mathrm{p} 50}$ and effectiveness.

\section{Experimental}

A general view of the cyclone type classifier that we call an accelerating cyclone vortex (ACV) is shown in Figure 1(a). The initial parameters of the main air flow are repre-

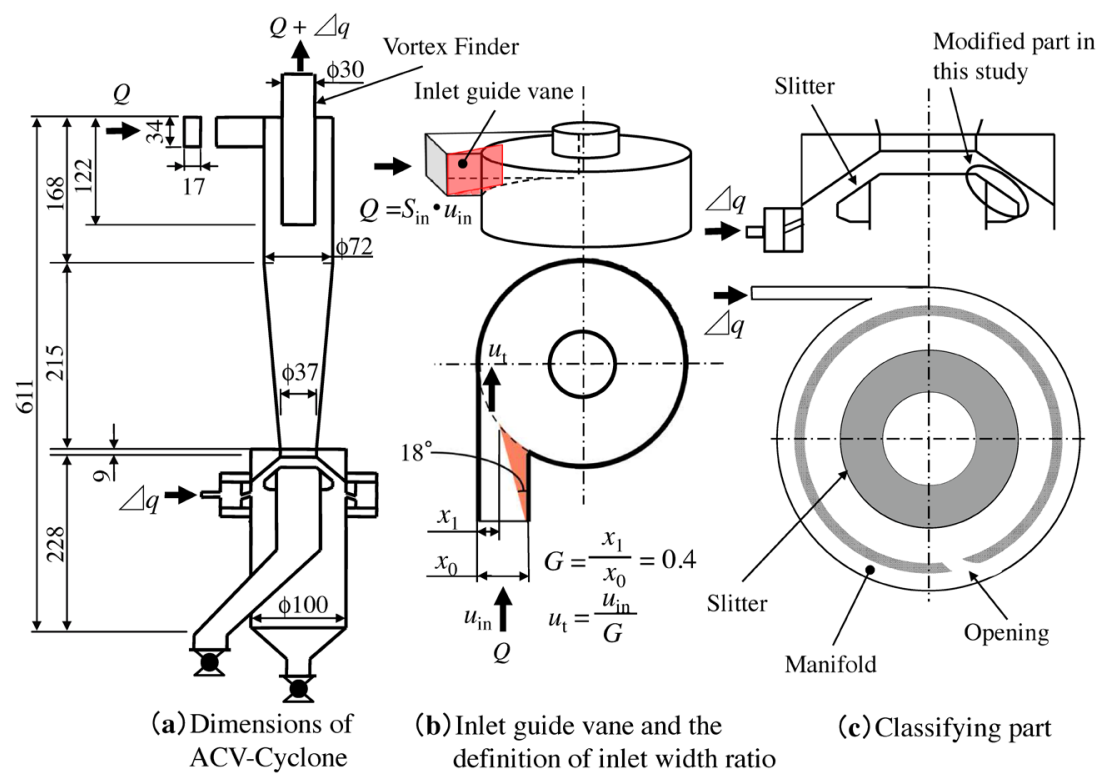

Fig. 1 Structure of the ACV-cyclone and dimensions of the main part 


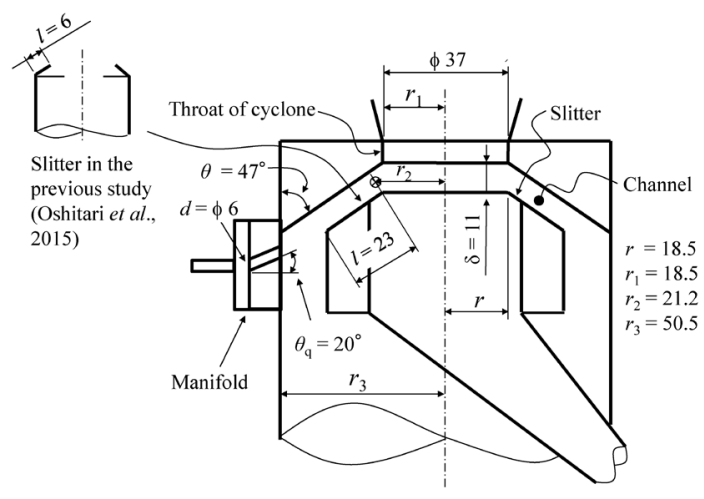

Fig. 2 Dimensions of the classifying part

sented by the flow rate $Q$ and the blow-up flow $\Delta q$. Each dimension is an internal value, and all dimensions are the same as those in the previous study (Oshitari et al., 2015) except for the length of the slitter. Figure 1(b) shows the inlet guide vane including the definition of the inlet width ratio $G$ at the entrance of the $\mathrm{ACV}$. The guide vane increases the velocity of the inlet air, allowing the tangential velocity of the air and particles to increase in the vicinity of the wall of the ACV. The experiment was performed with the ratio $G=0.4$, which showed the best classification effectiveness during the previous study. The value of $G$ was examined by Takeda et al. (2014), and they concluded that the most suitable value of $G$ is 0.4 from the relation between the pressure loss and the effect on the tangential velocity of a particle. Figure 1(c) shows a plane and side view of the classification section as well as the structure of the introduction section with the blow-up flow.

Figure 2 shows an enlarged view of the classification section consisting of the inner and outer collection boxes. The upper section of the inner box is a circular truncated cone shape, called the slitter, and an important section used for separating particles. The slope length $l$ of the slitter was increased to $23 \mathrm{~mm}$, while the length was $6 \mathrm{~mm}$ (Figure 2) in the previous study (Oshitari et al., 2015). The upper surface of the slitter forms the channel that separates the particles into the outer collection box. The radius $r_{2}$ is the distance from the center of the cyclone to the middle point of the separation channel based on the width at the tip of the slitter. Additional air was driven through the nozzle with a setting angle $\left(\theta_{\mathrm{q}}\right)$ of $20^{\circ}$ and diameter $(d)$ of $6 \mathrm{~mm}$. The blow-up flows into the outer collection box in the tangential direction after collecting in the manifold.

The fundamental principle of classification is shown in Figure 3. The particles are first supplied from the inlet, enter the channel along with the air of velocity $u_{\theta}$ by passing through the throat of the cyclone, and rotate at a velocity $v_{\theta}$ along the wall. Then, the particles are separated according to their size by the tip of the slitter. The larger particles on which the centrifugal force $F_{\mathrm{c}}$ acts with greater intensity relative to the drag force $F_{\mathrm{d}}$, are collected in the outer box. In contrast, the smaller particles, with the reverse relation, are collected in the inner box. $F_{\mathrm{c}}$ and $F_{\mathrm{d}}$ are induced by the

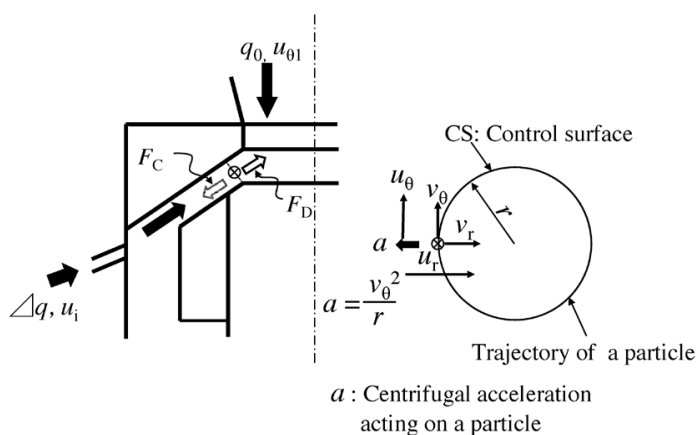

Fig. 3 Schematic diagram of the principle of separation

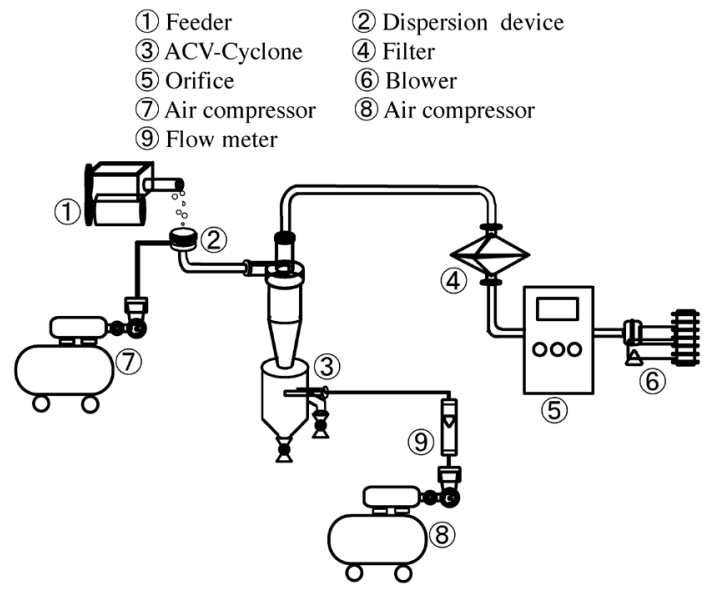

Fig. 4 Experimental flow diagram of the apparatus

acceleration $a$ and the relative velocity of the air $u_{\mathrm{r}}$ and the particle velocity $v_{\mathrm{r}}$, respectively. Hereafter, $u$ and $v$ denote the velocity of air and a particle, respectively, while the subscript, which is added as necessary, denotes the direction or the position. The subscripts $\theta$ and $r$ denote tangential and radial directions, respectively.

Figure 4 shows the arrangement of the experimental device. The particles were supplied at a constant rate by the feeder (1), and dispersed using a ring nozzle type disperser (Yamada et al., 1994). The blow-up air from the compressor 8 was driven into the outer collection box at a constant flow rate by the flow meter (9). The filter (4) was located at a point after the ACV to obtain the precise material balance. The particle size distribution was measured using a laser diffraction-type instrument (LA-950 manufactured by Horiba Co., Ltd.). The spherical acrylic particles, which were used for our classification, had a size distribution as shown in Figure 5. The experiments were performed under various operating conditions defined by $Q$ and $\Delta q$. The particles were classified in $5 \mathrm{~min}$ at a feed rate of $2 \mathrm{~g} / \mathrm{min}$. Additionally, the weight and size distributions of the particles that had collected in the collection boxes were measured for each experimental condition.

The classification performance was evaluated using the partial separation efficiency $\Delta \eta$, which is defined as follows. 


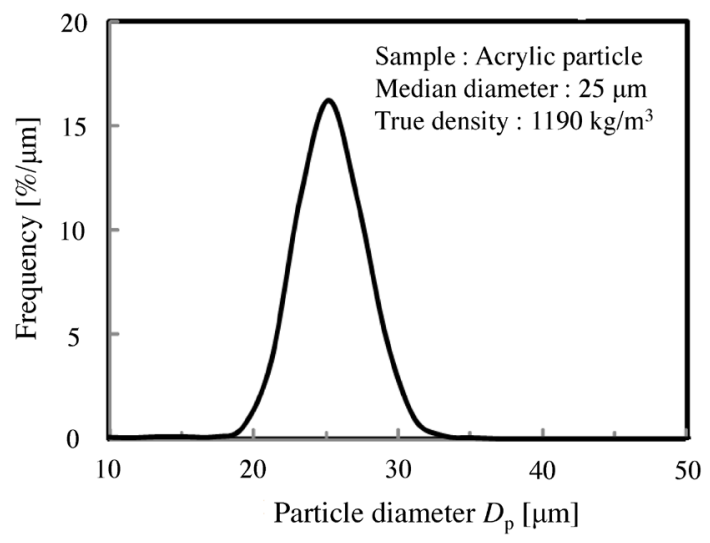

Fig. 5 Particle size distribution of the test powder

$$
\Delta \eta=\frac{m_{\mathrm{c}} f_{\mathrm{c}} \Delta D_{\mathrm{p}}}{m_{\mathrm{c}} f_{\mathrm{c}} \Delta D_{\mathrm{p}}+m_{\mathrm{F}} f_{\mathrm{F}} \Delta D_{\mathrm{p}}}
$$

The yield $\left(E_{\mathrm{c}}\right)$ of the coarse particles collected in the outer collection box is defined as follows.

$$
E_{\mathrm{c}}=\frac{m_{\mathrm{c}}}{m_{\mathrm{c}}+m_{\mathrm{F}}}
$$

Here, $m_{\mathrm{c}}$ and $m_{\mathrm{F}}$ represent the mass of the particles collected in the outer and inner collection boxes, respectively, and $f_{\mathrm{c}} \Delta D_{\mathrm{p}}$ and $f_{\mathrm{F}} \Delta D_{\mathrm{p}}$ are the mass fractions for the range of diameters from $D_{\mathrm{p}}-\Delta D_{\mathrm{p}} / 2$ to $D_{\mathrm{p}}+\Delta D_{\mathrm{p}} / 2$ in each of the boxes, respectively.

The following equation of Eq. (3) (Dirgo and Leith, 1985) was used as the most suitable fitting curve for $\Delta \eta$ since we had to calculate $\Delta \eta$ for $D_{\mathrm{p} 25}, D_{\mathrm{p} 50}$, and $D_{\mathrm{p} 75}$ from a limited number of experimental relationships between $\Delta \eta$ and $D_{\mathrm{p}}$.

$$
\Delta \eta=\frac{1}{1+\left(\frac{D_{\mathrm{p} 50}}{D_{\mathrm{p}}}\right)^{n}}
$$

Here, the values of $D_{\mathrm{p} 50}$ and $n$ were determined by the least squares method such that the result from Eq. (3) matched with the measured value.

\section{Results and Discussion}

\subsection{Effect of blow-up flow rate $\Delta q$ on $D_{p 50}$}

Figures 6, 7, and 8 show the partial separation efficiency curves for different values of the blow-up flow rate $\Delta q$ under constant main flow rates $(Q)$ of $600 \mathrm{~L} / \mathrm{min}, 800 \mathrm{~L} / \mathrm{min}$ and $900 \mathrm{~L} / \mathrm{min}$ respectively. In each figure, several curves were omitted to avoid visual complexity. However, the values of $D_{\mathrm{p} 50}, n$, and $E_{\mathrm{c}}$ are listed completely in the tables. The curves (Figure 6) show that $D_{\mathrm{p} 50}$ changed from 23.7 to $24.3 \mu \mathrm{m}$ only, although $\Delta q$ was increased from 0 to $80 \mathrm{~L} / \mathrm{min}$. On the other hand, the effectiveness notably improved as $\Delta q$ increased because of the increase in $D_{\mathrm{p} 25}$ and the decrease in $D_{\mathrm{p} 75}$. An improvement in the effectiveness was also confirmed by the increase in the values of $n$. With the curves of $\Delta \eta$ under the condition of $Q=800 \mathrm{~L} / \mathrm{min}$ (Figure 7) and $900 \mathrm{~L} / \mathrm{min}$

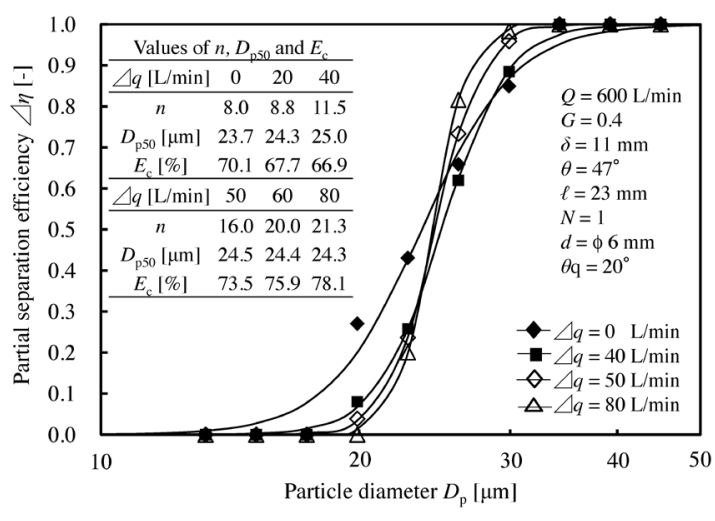

Fig. 6 Effect of the blow-up flow rate $\Delta q$ on classification performance

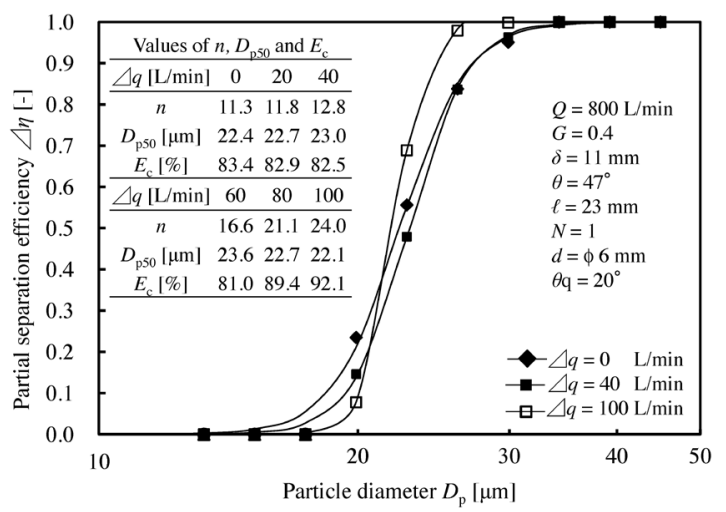

Fig. 7 Effect of the blow-up flow rate $\Delta q$ on classification performance

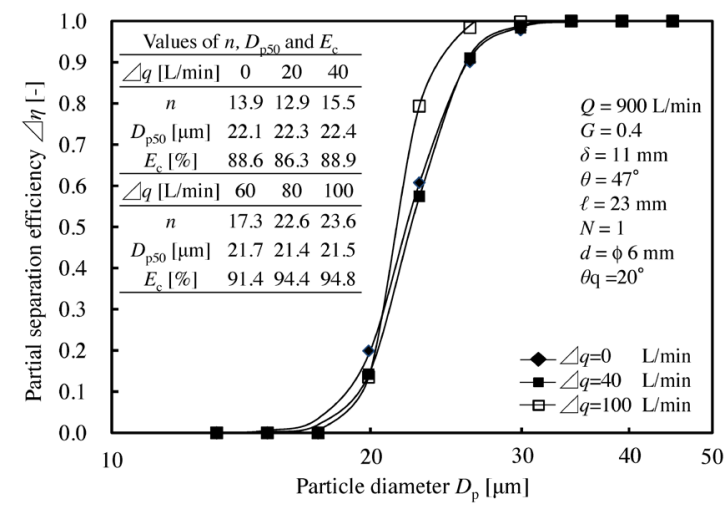

Fig. 8 Effect of the blow-up flow rate $\Delta q$ on classification performance

(Figure 8), the effect of $\Delta q$ on $D_{\mathrm{p} 50}$ and the effectiveness is approximately similar to as observed under the condition of $Q=600 \mathrm{~L} / \mathrm{min}$. Primarily, the effectiveness is improved with an increase of $\Delta q$ without $D_{\mathrm{p} 50}$ significantly changing, as will be discussed in detail.

Figure 9 shows the plots of $D_{\mathrm{p} 50}$ against $\Delta q$. In the figure, the values of $D_{\mathrm{p} 50}$ from our previous study $(l=6 \mathrm{~mm})$ were also plotted for comparison. The cut-off size $D_{\mathrm{p} 50}$ in this study $(l=23 \mathrm{~mm})$ is more or less constant. In contrast, $D_{\mathrm{p} 50}$ increased linearly in the previous study. Furthermore, other 
experiments, with $l=6 \mathrm{~mm}$, were performed. The curves of $\Delta \eta$ obtained from these experiments were similar to those obtained from experiments with $l=23 \mathrm{~mm}$. Therefore, it can be concluded that the channels of lengths greater than a certain value result in similar $\Delta \eta$ curves.

The classification model to estimate $D_{\mathrm{p} 50}$ reflects the classification mechanism. We consider the difference in $D_{\mathrm{p} 50}$ between this study and the previous study that used a particular classification model, and produced a new model to explain how $D_{\mathrm{p} 50}$ is determined for the long channel in this study. In our previous study, $D_{\mathrm{p} 50}$ was estimated using a static model where $D_{\mathrm{p} 50}$ was the diameter of the particle that was balanced by the centrifugal force and drag force at the CS. In that mathematical model, we used the tangential velocity of the airflow for expressing $D_{\mathrm{p} 50}$. However, in this study, we decided to directly use the velocity of the particle instead of that of air. Such a method provides insight into the classification mechanisms, and can explicitly relate the behavior of a particle to $D_{\mathrm{p} 50}$. The static model equation, which is obtained from Eq. (A1), based on $\mathrm{d} v_{\mathrm{r}} / \mathrm{d} t=0$ and $v_{\mathrm{r}}=0$ at the CS in the Appendix, is as follows.

$$
D_{\mathrm{p} 50}=\sqrt{\frac{18 \mu r_{2}}{\rho_{\mathrm{p}} S v_{\theta}^{2} \sin \theta}\left(\Delta q+q_{0}\right)}
$$

Here, $v_{\theta}$ is the tangential velocity of the particle at the CS and $q_{0}$ can be estimated based on results from our previous study (Oshitari et al., 2015) as $q_{0}=0.275 Q$. This key relationship and those obtained from the previous study are sum-

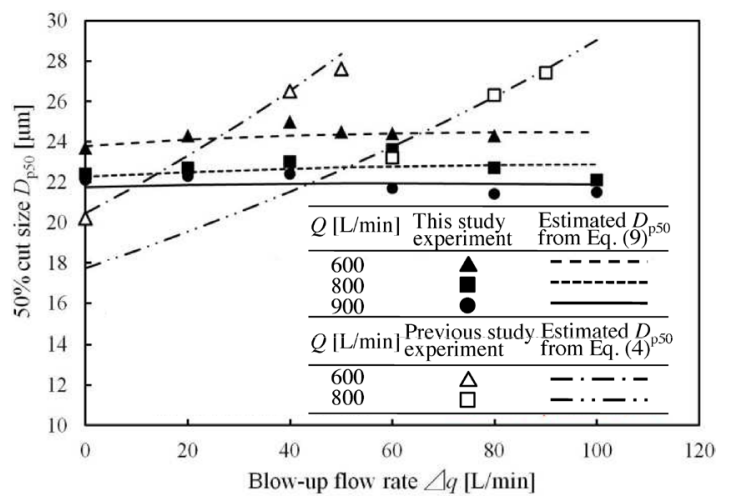

Fig. 9 Effect of blow-up flow rate $\Delta q$ on $D_{\mathrm{p} 50}$ marized and described in Table 1. If the tangential velocity of the particle for $\Delta q=0$ is designated as $v_{\theta 0}$, then $v_{\theta 0}$ can be expressed using the tangential velocity of the air $u_{\theta 1}$ at the throat of cyclone (Figure 2) as follows.

$$
v_{\theta 0}=K u_{\theta 1}
$$

Here, $K$ is the ratio of $v_{\theta 0}$ to $u_{\theta 1}$, and $u_{\theta 1}$ can be estimated from the relationship $u_{\theta 1}=1.03 u_{\mathrm{t}}$ (Table 1 ).

Meanwhile, we express the contribution of the blow-up flow for the particle tangential velocity $v_{\theta}$ as follows.

$$
v_{\theta}-v_{\theta 0}=A u_{\theta 3}
$$

Here, $u_{\theta 3}$ is the flow-out air velocity out of the blow-up flow nozzle and $A$ is the parameter related to the shape of the device. Combining Eq. (5), Eq. (6) and the relational expressions in Table 1 yields the following equation.

$$
\begin{aligned}
& v_{\theta}=v_{\theta 0}\left(1+\alpha \frac{\Delta q}{q_{0}}\right) \\
& \alpha=0.267 \frac{G S_{\text {in }} A}{K S_{\mathrm{i}}}
\end{aligned}
$$

Here, $\alpha$ is the parameter related to $A$ and $K$. The values of $K$ and $\alpha$ were obtained as 0.220 and -0.580 , respectively, by the least squares method such that Eq. (4) matched with previous experimental data on $D_{\mathrm{p} 50}$. In Figure 9, the dashdotted and dash-dot-dotted lines show the estimated $D_{\mathrm{p} 50}$, which almost coincides with the experimental $D_{\mathrm{p} 50}$. Then, Eq. (7) is reasonably accurate for evaluating the contribution of $q_{0}$ and $\Delta q$ to the particle tangential velocity $v_{\theta}$. The negative value of $\alpha(-0.580)$ indicates that the increase of the blow-up flow rate had weakened the tangential velocity $v_{\theta}$ while increasing $D_{\mathrm{p} 50}$, since the drag acting on the particle had become greater than the centrifugal force.

The classification results provided a small value for $K$, indicating that the particle had spiraled along the wall with a very small velocity relative to the air velocity. The reason is that the particle would have descended spirally through the boundary layer formed in the vicinity of the wall while colliding with the wall. This fact is supported by several studies

\begin{tabular}{|c|c|c|}
\hline No. & Relational expression & Description \\
\hline 1 & $q_{0}=0.275 Q$ & $\begin{array}{l}\text { The flow rate } q_{0} \text { can be estimated based on results from our previous study as } q_{0}=0.275 Q \text {. The value of } q_{0} \text { is the signifi- } \\
\text { cant air-flow rate that determines } D_{\mathrm{p} 50} \text { at } \Delta q=0 \text {. The air enters into the channel and exits with the same flow rate } q_{0} \text {. We } \\
\text { confirmed that the relational expression was completed with respect to } \delta \text { ranging from } 8 \text { to } 11 \mathrm{~mm} \text {, and } G \text { ranging from } \\
0.4 \text { to } 1.0 \text {. }\end{array}$ \\
\hline 2 & $\begin{aligned} u_{\theta 1} & =1.03 u_{\mathrm{t}} \\
& =1.03 \frac{Q}{G S_{\text {in }}}\end{aligned}$ & $\begin{array}{l}\text { The tangential velocity } u_{\theta 1} \text { can be estimated from the relationship } u_{\theta 1}=1.03 u_{\mathrm{t}} \text {, as was obtained from the tangential veloc- } \\
\text { ity distribution in the cyclone using the Meissner and Loffler }(1978) \text { model that has been reviewed by Cortés and Gil } \\
\text { (2007). The estimation needed the friction factor coefficient, which was assumed to be } 0.0058 \text { based on the study of Mus- } \\
\text { chelknautz and Trefz (1990). Here, } u_{\mathrm{t}} \text { is the air velocity at the cyclone entrance. The tangential velocity } u_{\theta 1} \text { is exactly equal } \\
\text { to the tangential velocity at the radius of the throat } r_{1} \text { in the tangential velocity distribution (Hoffmann and Stein, 2008). }\end{array}$ \\
\hline 3 & $u_{\theta 3}=\frac{\Delta q}{S_{\mathrm{i}}}$ & $\begin{array}{l}\text { The velocity } u_{\theta 3} \text { is the flow-out air velocity from the blow-up flow nozzle. The contribution of blow-up flow for the tan- } \\
\text { gential velocity of particles can be estimated based on } u_{\theta 3} \text {. }\end{array}$ \\
\hline
\end{tabular}
that involve the measurement of particle velocity near the wall of a cyclone separator (Li et al., 2009) and air-solid two-phase flow through a bend (Tanaka et al., 1988). It is

Table 1 Relational expression and description to use for the estimtion of $D_{\mathrm{p} 50}$ (Oshitari et al., 2015) 
also supported by the theoretical analysis of collision (Sommerfeld, 2003). According to the analysis, it is inferred that the value of $K$ is influenced by particle-fluid (air), particlewall, and particle-particle interaction in the vicinity of the cyclone wall. Furthermore, smaller diameter particles have a higher tangential velocity due to a lower frequency of collision with the wall. The value of $K$ primarily depends on particle diameter, particle density, and particle concentration concerning the particle viewpoint. Here, we adopted the same value of $K(0.220)$ that we had obtained in the above case, while considering the following.

Meanwhile, this study provided results that indicate that $D_{\mathrm{p} 50}$ hardly changes with the blow-up flow rate. The difference in $D_{\mathrm{p} 50}$ between the studies means that the particles, which entered the channel, were classified through another classifying mechanism. In order to explain this, a new classification model is necessary. Figure 10 shows schematically the comparison between the behavior of the particle in the new model (dynamic model) and the previous model (static model). In the static model, the particles are instantaneously classified at the tip of the slitter; in the dynamic model, the particles, which enter the channel, turnaround and approach the tip of the slitter, where the particles are classified. The equation of the dynamic model is obtained from Eq. (A1) with $\mathrm{d} v_{\mathrm{r}} / \mathrm{d} t \neq 0$ and $v_{\mathrm{r}}=0$ at CS. The mathematical model derived from the dynamic model is as follows. The derivation process is referred to in the Appendix.

$$
D_{\mathrm{p} 50}=\sqrt{\frac{18 \mu r_{2}}{\rho_{\mathrm{p}} S v_{\theta}^{2} \sin \theta}\left(\Delta q+\frac{q_{0}}{1-\exp \left(-q^{*} /\left(q_{0}+\Delta q\right)\right)}\right)}
$$

Here, the parameter $q^{*}$ is obtained from the experimental data $D_{\mathrm{p} 50}$ for the case where $\Delta q=0$ in Eq. (9). The parameter $q^{*}$ was determined by the least squares method; hence, Eq. (9) matched with the measured $D_{\mathrm{p} 50}$.

Figure 11 shows the experimental $D_{\mathrm{p} 50}$ and the fitted line obtained using Eq. (9). The $D_{\mathrm{p} 50}$ estimated from Eq. (9) coincides with the experimental $D_{\mathrm{p} 50}$. Additionally, the value of $q^{*}=218 \mathrm{~L} / \mathrm{min}$ was obtained from the fitted line. Figure 11 also shows the previous experimental $D_{\mathrm{p} 50}$ and the calculated $D_{\mathrm{p} 50}$ from Eq. (4), which were smaller and more sensitive to the main flow $Q$ than that of this study.

In this study, the value of $\alpha$ was obtained as 0.473 by the least squares method and by allowing the value of $D_{\mathrm{p} 50}$ from Eq. (9) to match with the experimental $D_{\mathrm{p} 50}$. The dashed lines and the solid line in Figure 9 show the estimated $D_{\mathrm{p} 50}$, which coincides with the experimental $D_{\mathrm{p} 50}$. In the Appendix, we assumed that $m=0.5$ holds regardless of the value of $\Delta q$ in the area of the CS, and $S=2 \pi r_{2} \delta m \sin \theta$. The assumption was confirmed on the basis of the level of consistency between the estimated $D_{\mathrm{p} 50}$ and experimental $D_{\mathrm{p} 50}$. The dynamic model accommodates the particle behavior and the classification mechanism for the channel length $l=23 \mathrm{~mm}$ in this study. The positive value $\alpha=0.473$ shows that the increase of the channel length led the blow-up flow to contribute highly to the increase in the particle tangential velocity $v_{\theta}$. The cut-off size $D_{\mathrm{p} 50}$ is decided by the main flow

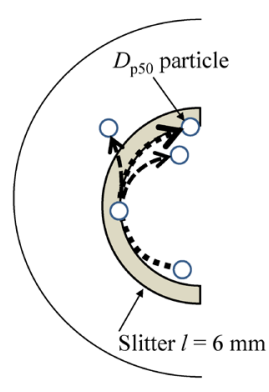

(a) Static model

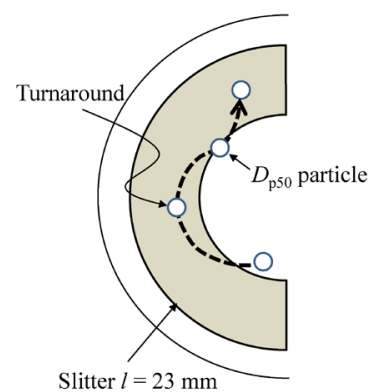

(b) Dynamic mode
Fig. 10 Schematic diagram comparing dynamic model with static model of classification

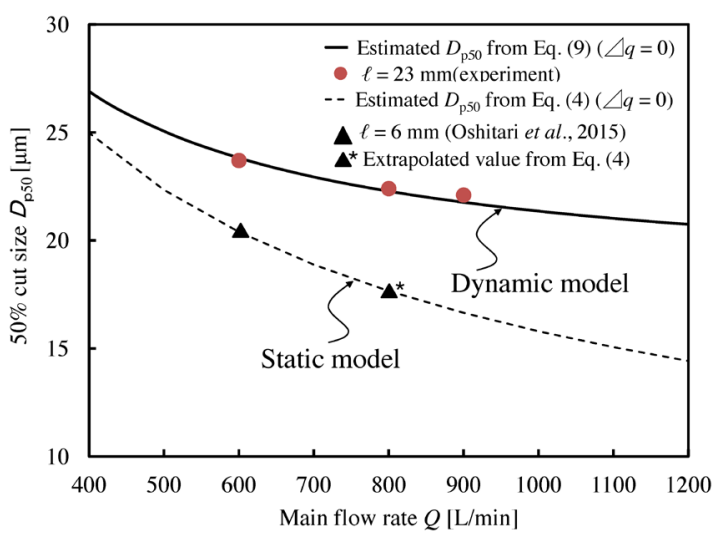

Fig. 11 Effect of main flow rate on $50 \%$ cut size $D_{\mathrm{p} 50}$

Table 2 Experimental-parameter values

\begin{tabular}{cccc}
\hline$l[\mathrm{~mm}]$ & $K[-]$ & $\alpha[-]$ & $q^{*}[\mathrm{~L} / \mathrm{min}]$ \\
\hline 6 & 0.220 & -0.580 & $\infty$ \\
23 & 0.220 & 0.473 & 218 \\
\hline
\end{tabular}

rate at the entrance regardless of the blow-up flow rate since the blow-up flow rate increases both the drag force and centrifugal force acting on the $D_{\mathrm{p} 50}$ particle.

We conclude that $t_{\mathrm{e}} / \tau$ is proportional to $1 /\left(q_{0}+\Delta q\right)$, where $t_{\mathrm{e}} / \tau$ is the dimensionless residence time turnaround point to the tip of the slitter in the channel in the Appendix. This fact was practically confirmed from the linearity of the relationship of $t_{\mathrm{e}} / \tau$ using Eq. (A5) and the values of $1 /\left(q_{0}+\Delta q\right)$. The experimental values of the parameters are listed in Table 2. Here, Eq. (9) is applicable to the static case since the dynamic model is reduced to Eq. (4) of the static model with $q^{*}=\infty$.

\subsection{Effect of blow-up flow rate $\Delta \boldsymbol{q}$ on effectiveness}

The effectiveness of classification can be evaluated using several methods depending on the requirements. We adopted $\kappa=D_{\mathrm{p} 25} / D_{\mathrm{p} 75}$, also called the sharpness index, which is generally used for evaluating the classification as well as the slope of $\Delta \eta$ curve at $D_{\mathrm{p} 50}$. Figure 12 shows each relationship of $\Delta q$ with $\kappa$ for $l=23 \mathrm{~mm}$ and $6 \mathrm{~mm}$. The value of $\kappa$ for $l=23 \mathrm{~mm}$ ranged from 0.75 to 0.92 for an increas- 


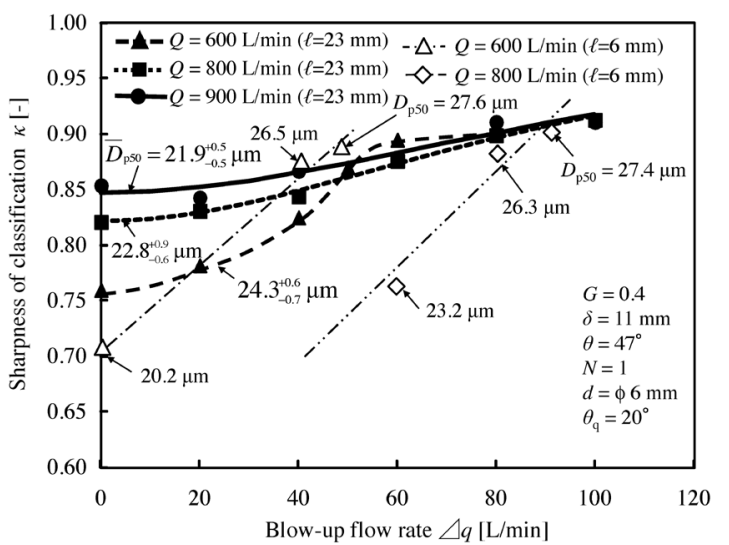

Fig. 12 Effect of blow-up flow rate $\Delta q$ on sharpness of classification

ing $\Delta q$. The curves for $Q=600 \mathrm{~L} / \mathrm{min}$ (Figure 6) show that initially, the increase in $\Delta q$ removes the fine particles from the channel by virtue of the drag force, and subsequently draws the coarse particles out by the centrifugal force into the outer collection box. Therefore, the value of $\kappa$ gradually increases because $D_{\mathrm{p} 25}$ increases to within the range of the lower $\Delta q$, and then, $\kappa$ sharply increases in the vicinity of $\Delta q=40 \mathrm{~L} / \mathrm{min}$ since $D_{\mathrm{p} 75}$ decreases. For $\mathrm{Q}=800$ (Figure 7) and $Q=900 \mathrm{~L} / \mathrm{min}$ (Figure 8), the measurable fine particles have already been removed even when $\Delta q=0$, and the value of $\kappa$ increases mainly due to the decrease in $D_{\mathrm{p} 75}$.

These characteristics show that the classification effectiveness is improved while holding the particle diameter relatively constant. The average $D_{\mathrm{p} 50}$ and the maximum deviation from the average with $l=23 \mathrm{~mm}$, and the value of $D_{\mathrm{p} 50}$ with $l=6 \mathrm{~mm}$ are denoted in Figure 12 to enable the comparison of the $\kappa$ values. The value of $\kappa$ ranged from 21.9 to $24.3 \mu \mathrm{m}$ for the average $D_{\mathrm{p} 50}$ is larger than that for the case where $l=6 \mathrm{~mm}$ when $\Delta q$ is above approximately $60 \mathrm{~L} / \mathrm{min}$.

In the previous channel, for $l=6 \mathrm{~mm}$, the increase in $\kappa$ was accompanied by an increase in $D_{\mathrm{p} 50}$ with an increasing $\Delta q$. This characteristic shows that the classification effectiveness was remarkably affected by the particle diameter. For $l=6 \mathrm{~mm}$, the value of $\kappa$ did not reach the same level as it did for the case of $l=23 \mathrm{~mm}$ until the cut-off size $D_{\mathrm{p} 50}$ was approximately $27 \mu \mathrm{m}$. This occurred since $\Delta q$ did not contribute to increasing the centrifugal force acting on the particles, only to eliminate them from the channel by the drag force in ascending order based on the particle diameter.

Several studies have shown that the values of $n$ are in the range of 2-4 (Hoffmann and Stein, 2008) for conventional cyclone separators, and 6.4 (Dirgo and Leith, 1985) for the most efficient separator. The value $\kappa=0.71$ is obtained using Eq. (3), where $n=6.4$, and $\Delta \eta=0.25$ for $D_{\mathrm{p} 25}$ and $\Delta \eta=0.75$ for $D_{\mathrm{p} 75}$. Considering the value $\kappa=0.71$, the ACV shows a considerably higher effectiveness than other cyclone separators.

Therefore, we identify the suitable operation condition using the dimensionless slope of $\Delta \eta$ as the appropriate effectiveness index. The slope is easily obtained as the dimensionless value $n / 4$ for the particular case of $D_{\mathrm{p} 50}$ by dif-

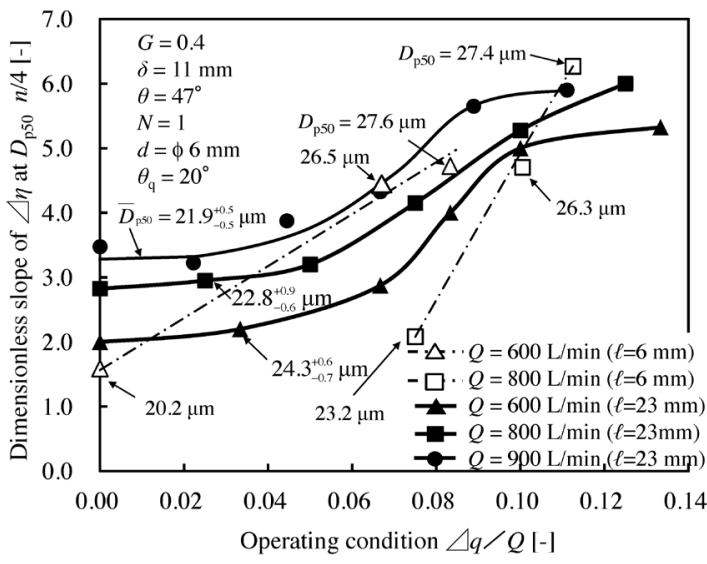

Fig. 13 Relationship between operating condition and classification effectiveness

ferentiating Eq. (3) with respect to $D_{\mathrm{p}} / D_{\mathrm{p} 50}$ and substituting $D_{\mathrm{p}} / D_{\mathrm{p} 50}=1$ into the differentiated equation. The relationship between the effectiveness index and the operation condition $\Delta q / Q$ is shown in Figure 13. This relationship is more straightforward than the relationship shown in Figure 12. The effectiveness index for the case of $l=23 \mathrm{~mm}$ sharply increased from approximately $\Delta q / Q=0.05$ to $\Delta q / Q=0.1$, indicating that the maximum effectiveness is accomplished when $\Delta q / Q$ is above approximately 0.1 .

While the increase in the $Q$ value resulted in a decrease in $D_{\mathrm{p} 50}$, it also increased the effectiveness. In contrast, the effectiveness index for the case of $l=6 \mathrm{~mm}$ linearly increased with an increasing $\Delta q / Q$. However, there is a natural limit since all particles are removed from the channel when $\Delta q / Q$ is too large. This operational condition should be determined from the relationship between the removing efficiency and the yield of a specified target-particle diameter. This was cited in the previous paper (Oshitari et al., 2015).

As shown above, $D_{\mathrm{p} 50}$ depends on the centrifugal and drag forces acting on particles. The ACV allowed an increase in $\Delta q$ to strengthen both the forces at the CS, thereby improving the classification effectiveness while holding $D_{\mathrm{p} 50}$ approximately constant. The ACV shows desirable characteristics, where particles with specified target-particle diameters are effectively classified without a decrease in the yield.

\section{Conclusions}

By classifying the acrylic fine particles with the developed cyclone type classifier (ACV), the following knowledge was obtained.

1. The cut-off size $D_{\mathrm{p} 50}$ is best accommodated by the dynamic model where the $D_{\mathrm{p} 50}$ particle turns around in the channel. Additionally, the $D_{\mathrm{p} 50}$ is determined in the non-equilibrium with respect to the centrifugal force and drag force at the CS.

2. The cut-off size $D_{\mathrm{p} 50}$ closely depends on the main flow rate $Q$ entering the entrance regardless of the blow-up flow rate $\Delta q$, since the increase in $\Delta q$ results in a simultaneous increase in the drag force and centrifugal force 
acting on the particle.

3. The classification effectiveness $\kappa=D_{\mathrm{p} 25} / D_{\mathrm{p} 75}$ was significantly improved by the increase in the blow-up flow rate. This increase in the blow-up flow rate resulted in an increasing $D_{\mathrm{p} 25}$ and decreasing $D_{\mathrm{p} 75}$. Consequently, the classifier was able to classify particles by $\kappa=0.75-0.92$. These particles would be collected, if conventional cyclones were used.

4. The relationship between the dimensionless slope at $D_{\mathrm{p} 50}$ and $\Delta q / Q$ permitted a clear evaluation indicating that the maximum classification effectiveness was accomplished when $\Delta q / Q$ is above approximately 0.1 .

\section{Appendix}

The motion of a particle in the channel was modeled by deriving the equation for estimating cut-off size $D_{\mathrm{p} 50}$ in terms of the air flow rate and the particle velocity as shown in Figures $\mathbf{A 1}(\mathbf{a})$ and $\mathbf{A 1}(\mathbf{b})$, respectively. The CS, which determines $D_{\mathrm{p} 50}$, was perpendicularly set to the slitter at the entrance of the channel. The radius of the CS is represented as $r_{2}$.

With respect to the flow of the air as shown in Figure A1(a), the air flows upward along the vortex slitter with a velocity of $u_{\mathrm{r}}+\Delta u_{\mathrm{r}}$. This is based on the situation where the air moves through the throat of the cyclone with a flow rate of $q_{0}$, and then turns around with the velocity $u_{\mathrm{r}}$ while the blow-up air flow rate $\Delta q$ contributes to the velocity $\Delta u_{\mathrm{r}}$ in the channel. As for the motion of the particle in Figure A1(b), the particle accompanying the air through the throat enters the channel and also turns around with the velocity $v_{\mathrm{r}}$, which is assumed to be equal to the air velocity $u_{\mathrm{r}}$. The particle then moves upwards along the vortex slitter with the air at a relative velocity $U^{\prime}=v_{\mathrm{r}}-\left(u_{\mathrm{r}}+\Delta u_{\mathrm{r}}\right)$.

Meanwhile, the particle velocity $v_{\mathrm{r}}$ decreases while moving along the slitter depending on the centrifugal acceleration $a=\left(v_{\theta}^{2} / r_{2}\right) \sin \theta$, which acts in reverse to the moving direction due to the particle rotation at velocity $v_{\theta}$ around the center of the cyclone. Here, the centrifugal acceleration $a$ and the rotating velocity $v_{\theta}$ are assumed to be constant and the rotating radius is represented by the radius of the CS $r_{2}$. For the conditions stated, $D_{\mathrm{p} 50}$ is considered as the diameter of the particle where the velocity $v_{\mathrm{r}}$ becomes zero at the CS.

A mathematical model for estimating $D_{\mathrm{p} 50}$ was derived

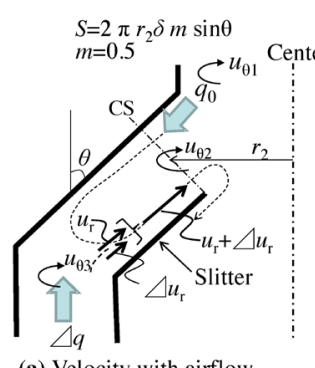

(a) Velocity with airflow

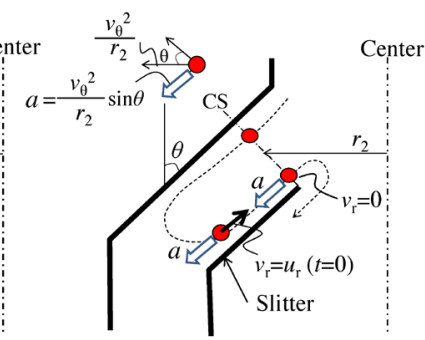

(b) Velocity and centrifugal force with a particle

Fig. A1 Schematic diagram indicating relative velocity with air and particle based on the equation of the particle motion. The equation, which has a one-dimensional coordinate taken in the direction along the slitter, is as follows.

$$
\left(\frac{\pi D_{\mathrm{p}}^{3}}{6}\right) \rho_{\mathrm{p}} \frac{\mathrm{d} U^{\prime}}{\mathrm{d} t}=-3 \pi D_{\mathrm{p}} \mu U^{\prime}-\left(\frac{\pi D_{\mathrm{p}}^{3}}{6}\right)\left(\rho_{\mathrm{p}}-\rho_{\text {air }}\right) a
$$

Here, $U^{\prime}$ is $v_{\mathrm{r}}-\left(u_{\mathrm{r}}+\Delta u_{\mathrm{r}}\right)$, and the particle velocity $v_{\mathrm{r}}$ is the only variable to depend on time. The first item on the right side is the drag force due to the airflow, and the second item is the body force induced by the centrifugal acceleration $a$. The application of Stokes' law of resistance provides the relationship $C_{\mathrm{D}}=24 / R e_{\mathrm{p}}$ where $R e_{\mathrm{p}}$ is defined as $R e=\rho U^{\prime} D_{\mathrm{p}} / \mu$.

These relationships were used to derive Eq. (A1).The true density of air, $\rho_{\text {air }}$ is neglected with respect to $\rho_{\mathrm{p}}$. Rearranging Eq. (A1) yields the following equation.

$$
\frac{\mathrm{d} U^{\prime}}{\mathrm{d} t}=-\frac{1}{\tau} U^{\prime}+a
$$

Here, $\tau$, called the relaxation time of the particle, is defined as follows.

$$
\tau \equiv \frac{D_{\mathrm{p}}^{3} \rho_{\mathrm{p}}}{18 \mu}
$$

Integrating Eq. (A2) for $t=0$ at the turnaround point, where the relative velocity $U^{\prime}$ is $\Delta u$ since $v_{\mathrm{r}}=u_{\mathrm{r}}$, provides the following equation.

$$
\left(u_{\mathrm{r}}+\Delta u_{\mathrm{r}}\right)-v_{\mathrm{r}}=\tau a\left(1-\exp \left(-\frac{t}{\tau}\right)\right)+\Delta u_{\mathrm{r}} \exp \left(-\frac{t}{\tau}\right)
$$

As described above, $D_{\mathrm{p} 50}$ is the particle diameter when $v_{\mathrm{r}}=0$ at the CS. In Eq. (A4), replacing $\tau$ by Eq. (A3), $D_{\mathrm{p}}$ by $D_{\mathrm{p} 50}, v_{\mathrm{r}}$ by zero, time $t$ by time $t_{\mathrm{e}}$, (i.e.. the time necessary for a particle to move from the turnaround point to the CS), $a$ by $v_{\theta}{ }^{2} \sin \theta / r_{2}, u_{\mathrm{r}}$ by $q_{0} / S$, and $\Delta u_{\mathrm{r}}$ by $\Delta q / S$, and then rearranging Eq. (A4) with $D_{\mathrm{p} 50}$ yields the following equation.

$$
D_{\mathrm{p} 50}=\sqrt{\frac{18 \mu r_{2}}{\rho_{\mathrm{p}} S v_{\theta}^{2} \sin \theta}\left(\Delta q+\frac{q_{0}}{1-\exp \left(-t_{\mathrm{e}} / \tau\right)}\right)}
$$

Here, $S$ is the area of the CS, which can be calculated as $2 \pi r_{2} \delta m \sin \theta$ where $m=0.5$ (half of the cross section of the channel). When the blow-up flow rate $\Delta q=0$, the rate of outflow from the channel is equal to the flow rate $q_{0}$. If the flow entering the channel does not significantly lose its kinetic energy in the channel, $m=0.5$ is obtained. Additionally, if the kinetic energy is not altered by the blow-up flow, $m$ remains at 0.5 . The assumptions are to be confirmed on the basis of the level of consistency between the estimated $D_{\mathrm{p} 50}$ and experimental $D_{\mathrm{p} 50}$. The flow rate $q_{0}$ and $\Delta q$ are transformed to the velocity $u_{\mathrm{r}}$ and $\Delta u_{\mathrm{r}}$, respectively, within the area $S$.

We concluded that $t_{\mathrm{e}} / \tau$ is proportional to $1 /\left(q_{0}+\Delta q\right)$, where $t_{\mathrm{e}} / \tau$ is the dimensionless residence time turnaround point to the tip of the slitter in the channel. Ultimately, we obtained the following equation. 


$$
D_{\mathrm{p} 50}=\sqrt{\frac{18 \mu r_{2}}{\rho_{\mathrm{p}} S v_{\theta}^{2} \sin \theta}\left(\Delta q+\frac{q_{0}}{1-\exp \left(-q^{*} /\left(q_{0}+\Delta q\right)\right)}\right)}
$$

Here, $q^{*}$ is assumed to be a constant parameter within our experimental conditions and with dimensions of flow rate. The parameter $q^{*}$ is the value of $q_{0}+\Delta q$ corresponding to $t_{\mathrm{e}} / \tau=1$. Additionally, the value of $1-\exp \left(-q^{*} /\left(q_{0}+\Delta q\right)\right)$ indicates the attainment level towards the equilibrium point, where the drag force is equal to the centrifugal force. The parameter $q^{*}$ is obtained from the experimental data $D_{\mathrm{p} 50}$ for the case where $\Delta q=0$ in Eq. (A6).

\section{Nomenclature}

$a=$ centrifugal acceleration

$C_{\mathrm{D}}=$ drag coefficient in Stokes' law

$d \quad=$ inside diameter of the blow-up nozzle

$D_{\mathrm{p}} \quad=$ particle diameter

$D_{\mathrm{p} 25}=25 \%$ cut-off size of the partial separation efficiency curve [m]

$D_{\mathrm{p} 50}=50 \%$ cut-off size of the partial separation efficiency curve [m]

$D_{\mathrm{p} 75}=75 \%$ cut-off size of the partial separation efficiency curve [m]

$E_{\mathrm{c}} \quad=$ yield of coarse particles

$F_{\mathrm{C}} \quad=$ centrifugal force induced by vortex flow

$F_{\mathrm{D}} \quad=$ drag force induced by main flow and blow-up flow

$f_{\mathrm{c}}\left(D_{\mathrm{p}}\right)=$ frequency of coarse particle size distribution

$f_{\mathrm{F}}\left(D_{\mathrm{p}}\right)=$ frequency of fine particle size distribution

$\left[\mathrm{m}^{-1}\right]$

$K=$ ratio of $v_{\theta 0}$ to $u_{\theta 1}$

$l=$ length of slitter

$M \quad=$ ratio of control surface (CS) area to cross section of channel

$\left[\mathrm{m}^{-1}\right]$

$[-]$

$[\mathrm{m}]$

$m_{\mathrm{F}}, m_{\mathrm{c}}=$ mass of total particles within the inside and outside collection boxes

$N=$ number of blow-up nozzles

$=$ exponent in Eq. (3)

$Q \quad=$ rate of inlet air flow into cyclone (primary flow rate)

$=$ flow rate of air into channel through throat of

$[\mathrm{L} / \mathrm{min}]$ or $\left[\mathrm{m}^{3} / \mathrm{s}\right]$ cyclone

$[\mathrm{L} / \mathrm{min}]$ or $\left[\mathrm{m}^{3} / \mathrm{s}\right]$

$q^{*}$

$=$ constant parameter relative to $t_{\mathrm{e}} / \tau=1$ in Eqs. (9) and (A6)

$\left[\mathrm{m}^{3} / \mathrm{s}\right]$

$=$ radius of tip of slitter from center of cyclone

$[\mathrm{m}]$

$=$ radius of throat of cyclone

$=$ representative radius of CS from center of cyclone

$=$ radius of outside collection box

$=$ area of CS

residence time from turnaround point to CS in Eq. (A5) [s]

$=$ velocity within blow-up nozzle

$=$ velocity of air flow at cyclone inlet

$=$ velocity of air flow along channel

$=$ velocity of converged air flow by inlet guide vane

$=$ tangential velocity of air

$=$ tangential velocity of air in throat of cyclone

$=$ tangential velocity of $q_{0}+\Delta q$

$=$ tangential velocity of blow-up flow rate $\Delta q$

$=$ velocity of particle along channel

$=$ tangential velocity of particle at CS

$=$ tangential velocity of particle with $\Delta q=0$ at CS of particle

$[-]$

$\Delta q \quad=$ blow-up flow rate of air $\quad[\mathrm{L} / \mathrm{min}]$ or $\left[\mathrm{m}^{3} / \mathrm{s}\right]$

$\Delta u_{\mathrm{r}}=$ velocity of air induced by blow-up flow along channel $[\mathrm{m} / \mathrm{s}]$

$\Delta \eta \quad=$ partial separation efficiency [-]

$\delta \quad=$ vertical width of channel $\quad[\mathrm{m}]$

$\theta=$ inclination angle of slit [degree]

$\theta_{\mathrm{q}}=$ inclination angle of blow-up nozzle [degree]

$\kappa=$ classification sharpness index $\left(=D_{\mathrm{p} 25} / D_{\mathrm{p} 75}\right) \quad[-]$

$\mu \quad=$ viscosity of air $\quad[\mathrm{Pa} \cdot \mathrm{s}]$

$\rho_{\text {air }}=$ true density of air $\quad\left[\mathrm{kg} / \mathrm{m}^{3}\right]$

$\rho_{\mathrm{p}} \quad=$ true density of particle $\quad\left[\mathrm{kg} / \mathrm{m}^{3}\right]$

$\tau \quad=$ relaxation time of particle $\quad[-]$

\section{Literature Cited}

Akiyama, S., H. Yoshida, K. Fukui, K. Ono, R. Nobukiyo and Y. Inada; "Improvement in Classification Performance of Gas-Cyclone by Local Fluid Velocity Control," J. Soc. Powder Technol., 42, 401-408 (2005)

Barth, W.; "Design and Layout of the Cyclone Separator on the Basis of New Investigations," Brennstoff-Warme-Kraft, 8, 1-9 (1956)

Cortés, C. and A. Gil; "Modeling the Gas and Particle Flow Inside Cyclone Separators," Pror. Energy Combust. Sci., 33, 409-452 (2007)

Dirgo, J. and D. Leith; "Performance of Theoretically Optimized Cyclone," Filtr. Sep., 22, 119-125 (1985)

Hoffmann, A. C. and L. E. Stein; "Gas Cyclone and Swirl Tubes Principle, Design and Operation,” pp. 183-192 2nd ed. Springer, Berlin Heidelberg, Germany (2008)

Iinoya, K., T. Fuyuki, Y. Yamada, H. Hisakuni and E. Sue; "Dry Submicron Classification by a Small Blow Down Cyclone," J. Soc. Powder Technol, 29, 351-355 (1992)

Li, S., H. Yang, H. Zhang, S. Yang, J. Lu and G. Yue; "Measurement of Solid Concentration and Particle Velocity Distributions Near the Wall of a Cyclone," Chem. Eng. J., 150, 168-173 (2009)

Meissner, P. and F. Loffler; "For Calculation of the Flow Field in Cyclone Separator," Chem-Ing-Tech., 50, 471 (1978)

Muschelknautz, E. and M. Trefz; "Design and Calculation of Higher and Highest Loaded Gas Cyclones," Proceedings of Second World Congress on Particle Technology, pp. 52-71 Kyoto, Japan (1990)

Oshitari, T., M. Kimura, K. Yamamoto, K. Fukui and H. Yoshida; "Development and Evaluation of a Cyclone Type Classifier for Separating Coarse Particles," J. Soc. Powder Technol. Japan, 52, 435-444 (2015)

Reydon, R. F. and W. H. Gauvin; "Theoretical and Experimental Studies in Combined Vortex Flow," Can. J. Chem. Eng., 59, 14-23 (1981)

Sommerfeld, M.; "Analysis of Collision Effects for Turbulent Gas-Particle Flow in a Horizontal Channel: Part 1. Particle Transport," Int. J. Multiph. Flow, 29, 675-699 (2003)

Takeda, N., T. Oshitari, T. Yamamoto, K. Fukui and H. Yoshida; "Effect of Inlet Clean Air and Guide Plate on Fine Particle Classification of Gas-cyclone," J. Soc. Powder Technol. Japan, 51, 614-622 (2014)

Tanaka, T., H. Ishibashi, T. Shiratori, Y. Tuji and Y. Morikawa; "AirSolid Two-Phase Flow through a Bend (Flow Patterns and Particle Velocity (in Japanese)," Transactions of JSME, 54, 367-373 (1988)

Yamada, Y., H. Murata, E. Shinoda and K. Iinoya; "Dispersion Performance of Dry Powder by Ring Nozzle Jet," Kagaku Kogaku Ronbunshu, 20, 352-359 (1994)

Yoshida, H., T. Saeki, T. Fujioka, T. Ueda and T. Fuyuki; "Fine Particle Separation by Revised Air-Cyclone Classifier," Kagaku Kogaku Ronbunshu, 19, 476-482 (1993)

Yoshida, H., T. Fujioka, K. Hashimoto and K. Nagahashi; "Effect of Blow-Down on Fluid and Particle Movement in Cyclone Classifier," Kagaku Kogaku Ronbunshu, 21, 594-602 (1995) 